\title{
Study on a Dynamic E-business Application Framework Based on Web Service-Based SOA
}

\author{
Xueqiang Yin \\ Department of Public Safety, China Electronic Technology Group Corporation \\ No.15 Research Institute, Beijing 100083 China
}

\begin{abstract}
Web service is the kernel and key to develop a new dynamic E-business application framework and the dynamic E-business is the goal of E-business development. This paper introduced several key techniques of XML, SOAP, WSDL and UDDI in Web services as well as the relationships between these techniques. And the SOA can meet the requirements of various kinds of E-business application framework. So a service-oriented dynamic E-business application framework based on SOA and Web service is proposed. In the proposed framework, SOA is used to realize the standard and loosely coupled application framework by using the characteristics of Web service. They can ensure a compatible information exchange and integration framework for the business process of the dynamic E-business, provide more complex interaction and collaboration between trading partners. So the proposed E-business application framework has these characteristics of crossing platform, flexibility and easy expansion, and can effectively solve dynamic E-business application framework.
\end{abstract}

Keywords: Dynamic E-business; application framework; Web service; SOA; platform

\section{Introduction}

Internet is changing each part in our lives. The most fast and efficient E-business integration can put up a close connection among the enterprise, manufacturers and customers. It can provide a simple communication method and significant economic returns. With the continuous development of E-business, the focus of E-business is gradually moved from the initial $\mathrm{B} 2 \mathrm{C}$ to more challenging $\mathrm{B} 2 \mathrm{~B}[1]$. In order to let enterprises to obtain highly efficient business, increase the income and reduce costs for getting greater business and competitive returns, the designers of E-business solution scheme need consider crossing different network configuration from the various private internal network of enterprises to Extranet of sharing information data among them[2].

At present, the lot of operation modes of E-business depends on disunity infrastructure, which result in the different contact information among the buyer, supplier, market and service providers. So it is extremely difficult to realize the information exchange, data integration and application integration by using different business entities of underlying architecture. This will greatly limit to use Web for conducting a wider range of E-business cooperation. In recent years, many searchers proposed E-business application architectures. Flurry and Vicknair[3] described the IBM application framework for ebusiness (the Framework) based on enterprise Java ${ }^{\mathrm{TM}}$ technologies for achieving business transformation and a foundation framework for developing and extending e-business processes and applications. Rajiv et al.[4] proposed a customer relationship management (CRM) approach by a hospital through a web-based physician profiling system (PPS). Hasselbring[5] proposed a top-down approach to make them flexible, scalable, and useable, require systematic development for E-business applications. Wu et al.[6] proposed a service based e-business application integration framework (SEAIF), which is based on XML to integrate enterprise application and B2B e-business process. UDDI 
specification was enriched, and the basic elements of web service interoperation markup language were defined on the top of hierarchy of UDDI. Li et al.[7] proposed a the concept of e-union, which integrates the services provided by different e-trading construction sites to provide an open e-trading service. The design of a mobile agentenabled framework for building such an open e-trading marketplace environment is described. Al-HelaliAdnan and Zhang[8] proposed a secure framework that provides XML signature function, XML Element-wise Encryption function, smart card based crypto API library and Public Key Infrastructure (PKI) security functions. Benatallah[9] proposed a framework that builds on current standards to help developers define extended service models and richer Web service abstractions. The framework's main feature is a conversation metamodel derived from the authors' analysis of E-business portal sites. Cho et al.[10] proposed a recommendation methodology based on Web usage mining, and product taxonomy to enhance the recommendation quality and the system performance of current CF-based recommender systems. Schmid and Rossi[11] proposed a design approach that improves earlier work and models business processes explicitly along with navigation elements. Zhang et al.[12] proposed proposed a framework for dynamic and personalized composition of Web services using an approach that integrates not only functional attributes of Web services, but also nonfunctional attributes such as service requirements, quality of service, and the preferences and constraints of individual service consumers. Francisco et al.[13] proposed a framework that merges various advanced information technologies for developing electronic commerce (E-business) applications. The use of E-business utilities provides several advantages to businesses. March and Ngai[14] proposed a conceptual model to show how managers evaluate internal (relative advantage and compatibility of adopting an innovation) and external (competitive pressure and partner conflict) determinants that affect the intention to adopt technological innovations. Chou and Lee[15] proposed a research framework to describe the enterprise application integration (EAI). Then, two illustrations are used to explain the generality of our method. Li et al., [16] proposed a multi-model driven collaborative development platform for service-oriented e-Business systems. The platform provides engineers/consultants with three views, i.e., business view, process view, and service view to support service-oriented software engineering, top-down business design and bottomup service composite and development. Hsia et al., [17] proposed a goal-driven methodology for eliciting and modeling the requirements of a business-to-consumer application. This methodology enables business managers and system developers to develop high-level strategies that improve value activities and obtain competitive advantage, and thereby determine the specifications of the core e-services. Huang et al., [18] proposed a performance assessment model for E-business; it included indicators, indicator weights, and evaluation methods. The model has seven methods of assessing Ebusiness performance and uses four criteria to compare and select the appropriate one for a particular situation. Wei et al.[19] proposed a new business logic framework for modern E-business combined within the enterprise management system such as ERP and CRM, and integrated to the operation flow and distribution platform. Piao et al.[20] proposed the open application programming interfaces (APIs) from eBay and Taobao E-business websites and captured real transaction data. Pajek is used to visualize the agent relationships in the transaction network. Meer et al.[21] proposed a cost-based database request distribution (C-DBRD) strategy, a policy to distribute requests, across a cluster of commercial, off-the-shelf databases, and discuss its implementation. Chen and Zhang[22] introduced the ECRM and E-business strategy in enterprise and use VAR model to analyze how E-Business Application affects business performance in emerging countries like China. The results show that E-business application can improve the sales performance of small business, and E-business is an important factor to success. Zhang et al., [23] investigated two concept extraction methods: Automatic Concept Extractor (ACE) and Automatic Keyphrase Extraction (KEA). We upgrade ACE into Improved Concept 
Extractor (ICE) with significant improvements. We evaluate the methods by comparing automatically generated concepts to a gold standard. Wang and Lv[24] analyzed the influence factors of E-business application on the business model of Chinese real estate enterprises. Gao et al.[25] extended the classical finite state machine (FSM) to formalize the behavior of Web application, namely the extended FSM for Web applications (EFSM4WA) model. This model is also suitable to formally describe the interaction behaviors of dynamic reconfiguration when Web application encountered failure. Eugenio et al.[26] proposed an architectural pattern that helps designers to easily identify the subsystems that characterize intelligent enterprise systems. By decoupling transactional behavior from batch processing, the pattern avoids the interference of knowledge extraction and reasoning processes with the state and the performance of the transactional subsystem. Liu et al.[27] analyzed the definition of electronic commerce, which should be the most important business model with advanced technology of information. It also analyzed the use of economic commerce and accompanied interests through specific theories and actual cases. Lamprinakos et al.[29] described the design and implementation of such platform that enables the deployment of services to follow-up the patient's health status based on a set of monitored parameters per disease and to profile user's habits and diagnose deviations from their usual activities. Martínez-Carreras et al., [28] describes and detailed the elements for building the next generation of integrated business environments (IBE) and to analyze the features of ESBs as the core of this infrastructure.

The dynamic E-business is the development goal of E-business. Its features are to defer to business process modeling and design reused and flexile components. For the traditional technologies, there are quite prominent problems. The E-business lacks the uniform business publishing and mechanism to cause extremely difficult information exchange and cooperation. And the system development exists long development time, different reconstruction, difficult upgrade and maintenance. These shortcomings greatly limit the further development of E- business. However, Web service provides new method for realizing dynamic E-business application architecture. So dynamic Ebusiness application framework based on Web Service-based SOA is proposed in this paper. The main technologies are expatiated in detail.

The rest of this paper is organized as follows. Section 2 briefly introduces Serviceoriented architecture and Web service. Section 3 analyzed the traditional E-business application framework. Section 4 presents a new dynamic E-business application framework based on Web Service-based SOA. The basic principles and characteristics of dynamic E-business application framework are expatiated in detail in this Section. Finally, the conclusions are discussed in Section 5.

\section{Service-Oriented Architecture and Web Service}

\subsection{Service-Oriented Architecture}

Service-oriented architecture (SOA) is a solution to design and construct the loosing coupling software system, the SOA can publish the business functions by using programmable and accessible service. And other application can use these services by the published and findable interface. So the key concept of SOA is service and each application of SOA is taken as a service, which are called and administrated. W3C defined SOA: the service provider delivers ultimately results to service users by finishing a set of work. The last results usually change the status the status of user or/and provider. To a certain extent, the SOA framework is a model for designing, developing, deploying and administrating discrete logic units.

The Web service-Based SOA framework is shown in Figure 1. The framework includes three roles. Service provider publishes its own service and responses the request. Service broker registers and classifies the published service providers, it can provide 
search service. Service requester seeks requisite service by using service broker, and it can make use of the service. The components of SOA ought to have one or more of the mentioned roles. These roles main are "publish" operation, "find" operation and "bind" operation. Publish operation helps service provider to register its own function and interface. Find operation helps service requester to find special service by service broker. Bind operation helps service requester to use the provided services.

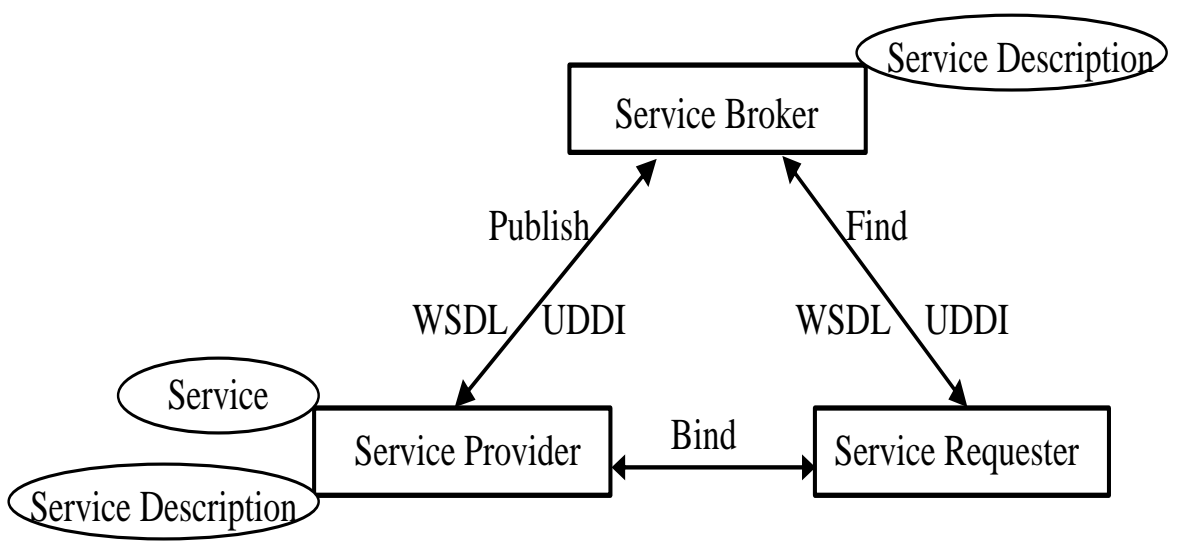

Figure 1. Web Service-Based SOA Framework

The basic task of SOA framework is service. The service lies in the layer between business requirement and bottom technology. The dynamic relations among function requirements, acquired services and bottom implemented technologies are understood in the process of designing software system based on Web service-based SOA framework. The basic service has to meet the requirement of definition definitude and function singularity in the SOA.

\subsection{Web Service}

Web service combines the component-oriented methods and Web technologies, it can better describes its own service. The functions of Web service are simple, but it includes extremely complex business logic. When Web service is deployed, the other applications can be found and requested. The key technologies of Web service are shown in Figure 2.

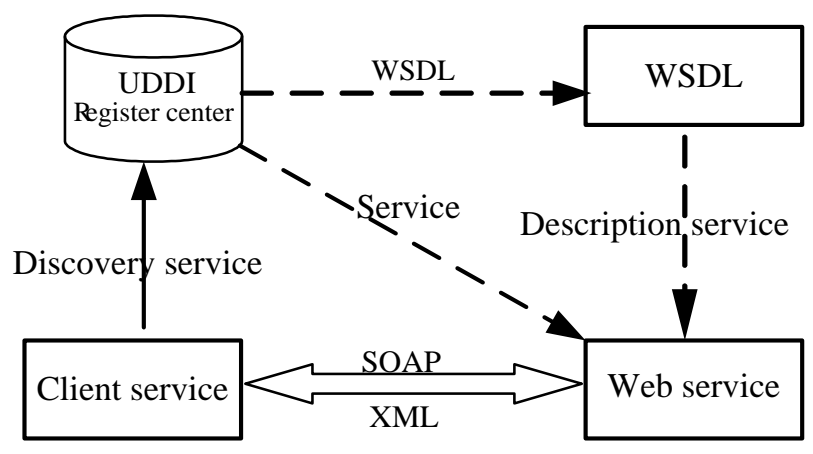

Figure 2. Key Technologies of Web Service

As is known to all, the service is the kernel of Web service. Web service is a kind of realization of SOA. In addition, the three roles of the SOA can only be executed when the components of SOA are interact. So a lot of standardized technologies are used to realize the application framework in Web service, these technologies mainly includes XML, SOAP, WSDL, UDDI, HTTP and so on. 


\section{(1) XML}

$\mathrm{XML}$ is Extensible Markup Language, and it is a markup language based on text W3C specification. It is a subset of the SGML for particular application field. XML strictly defines the structured data that can be transplanted. It is a key point of Web service that uses XML to express operation and data. And it gives the context dependent function to the structured data.

\section{(2) SOAP}

SOAP (Simple Object Access Protocol) is a simple communication protocol based on $\mathrm{XML}$ in the distributing environment. It is used to equivalently and conveniently exchange data according to the form of the object between the application programs. In the SOAP, HTTP is regarded as the communication protocol, RPC is regarded as the consistency call path, XML is regarded as the data transmission format. The service providers and service customers execute communication interaction on the Internet through the firewall in order to support the standard protocols of SMTP, FTP, TCP, POP3 and so on. SOAP is completely independent of any manufacturer. It can independently implement for any programming language, object model, operating system and platform.

\section{(3) WSDL}

WSDL (Web Service Description Language) is a XML standard by a lot of business associations, such as Microsoft, IBM and so on. WSDL could describe the Web service in detail by defining a standard XML syntax. The other applications can be given:

(a) How to find a special Web service

(b) How to communicate with a Web service (by using SOAP)

(c) How to generate a code to represent Web service

\section{(4) UDDI}

UDDI is a developed project by Microsoft, Arriba, IBM and other IT companies. The goal of UDDI is how to find Web service and question by using service. The UDDI is Universal Description, Discovery, and Integration. At present, the UDDI is becoming the standard for client applications in order to find Web service and publish its own Web service.

The provided information of UDDI business registry centre mainly includes the following contents:

\section{(a) White Page}

It provides these information of business name, business description, business contact and so on.

(b) Yellow Page

It provides the industry category information based on standard classification method.

(c) Green Page

It provides the technical information of Web Service about the business entity, that is, It is to describe the actual strategy, deal with business process and bind description ability.

\subsection{Features of Web Service-Based SOA}

2.3.1. Communication across Firewall: Web service can exchange provided data by using SOAP-based XML documents and common communication mode, for example, HTTP, FTP, and SMTP and so on. So the communication based on Web service can breezily permeate firewall. All devices with supporting HTTP and XML can access to Web service, and each Web service also can access to other Web service.

2.3.2. Easy Realization: There are a large number of free development tools, which are proved by IT companies to construct and deploy Web service, such as IBM and Microsoft and so on. At the same time, the JavaBeans and COM components can be easily translated into Web service in order to provide the service. 
2.3.3. Loosing Coupling: Web service takes on a loosing coupling framework. Each Web service can change its own running mechanism, but it can not affect other Web service. If the Web service is updated, the clients can call the service in order to obtain the updating results.

2.3.4. Reused Software: The codes can be reused in Web service, as well as data that behind the codes. These features of Web service-based SOA can expand Web service function, truly realize the mutual operation. And they may use the loosing coupling model to expand each kind of data and the service resource. In studying and designing the dynamic E-business application framework, a perfect solution is proved for existing and likely-to-appear problems.

\section{A Traditional E-Business Application Framework}

The E-business is developed by the following several steps. From the static web page, the interactive network tabulation is used and the enterprise to the client (B2C) and the enterprise to the enterprise (B2B) is executed. At present, many enterprises still use static web page and simple E-business application. The E-business technologies of EDI and EAI based on the complex application linking. The traditional E-business links the Ebusiness application, users and the additional information systems by using the codes. So the traditional E-business application framework is shown in Figure 3.

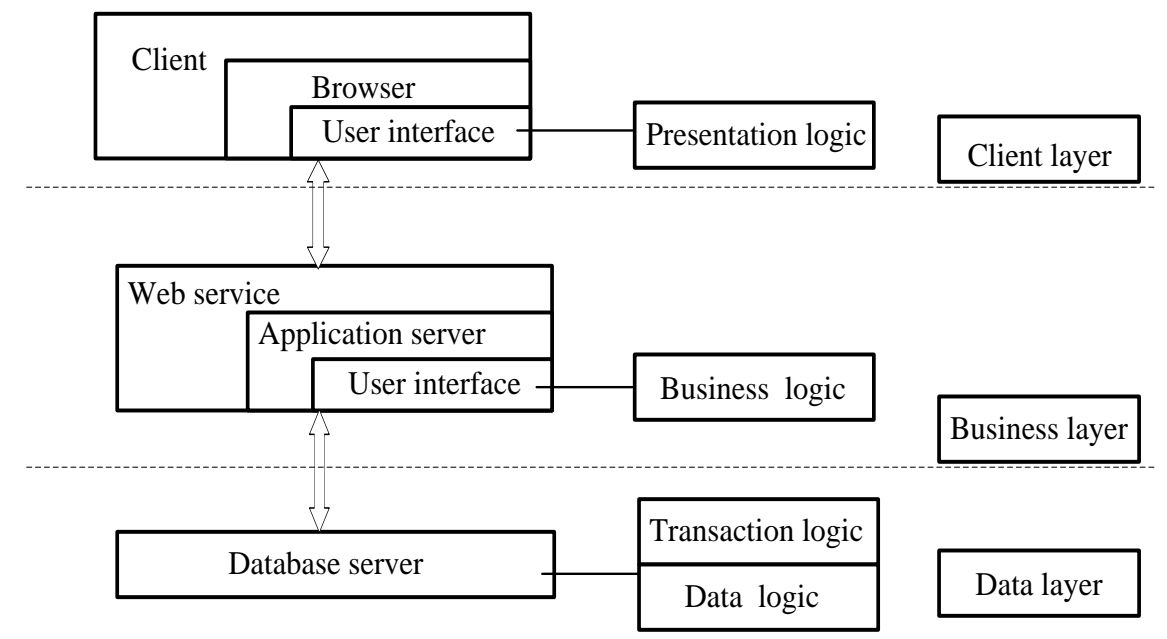

Figure 3. Traditional E-business Application Framework

\section{A New Dynamic E-Business Application Framework}

The traditional E-business application framework takes on poor integration ability, it can not validly solve larger expense for changing E-business flow and customize the demand of each category of users. So it is an optimal scheme to develop dynamic Ebusiness application framework for solving the above problems. The dynamic E-business is the next generation E-business based on emphasizing comprehension and infrastructure of B2B. The dynamic E-business can obtain the best benefit for the interior and exterior enterprises by adjusting the Internet standard and general infrastructure. The enterprises must construct the massive applications between the service and partners in B2B to realize the dynamic E-business. The application service can directly interact by using agreeing interaction or integrated protocol. A dynamic E-business application framework is shown in Figure 4. 


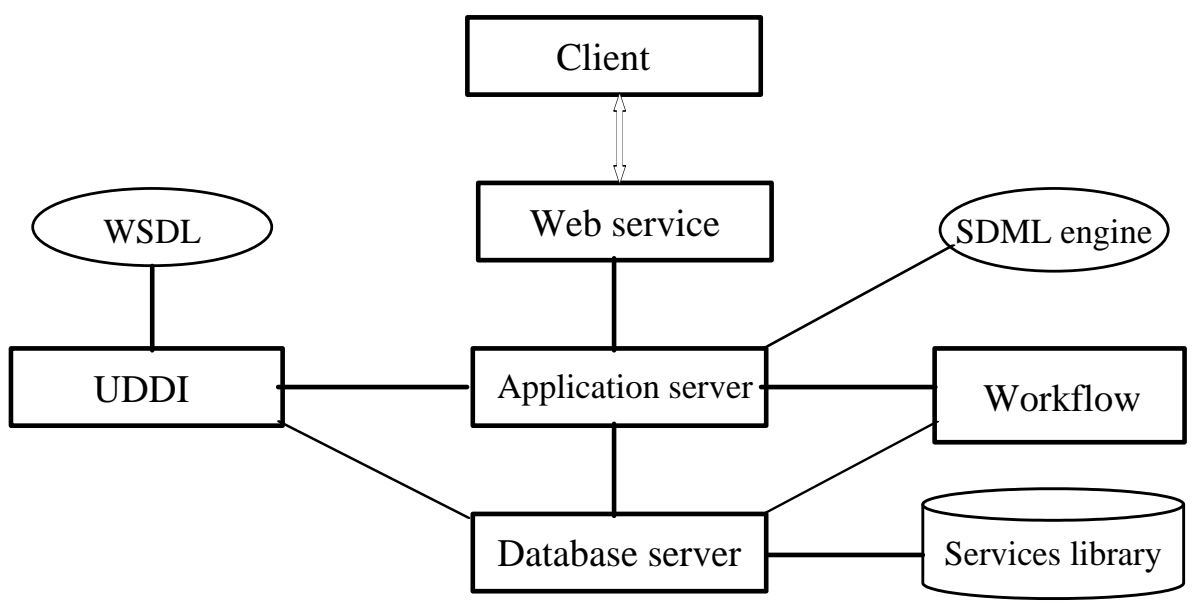

Figure 4. Dynamic E-business Framework

The dynamic E-business application framework takes on some advantages, such as the relative independent and convenient operation. At this time, it also takes on some disadvantages, such as too substantial investment, different data sharing, discommodious system upgrade and information exchange, long-distance designing development time and so on. So a dynamic E-business application framework based on Web service-based SOA is proposed, shown as Figure 5. The dynamic E-business application framework can automatically, immediately find the correlation application of the different commercial entities on Internet in order to greatly meet the needs of clients.

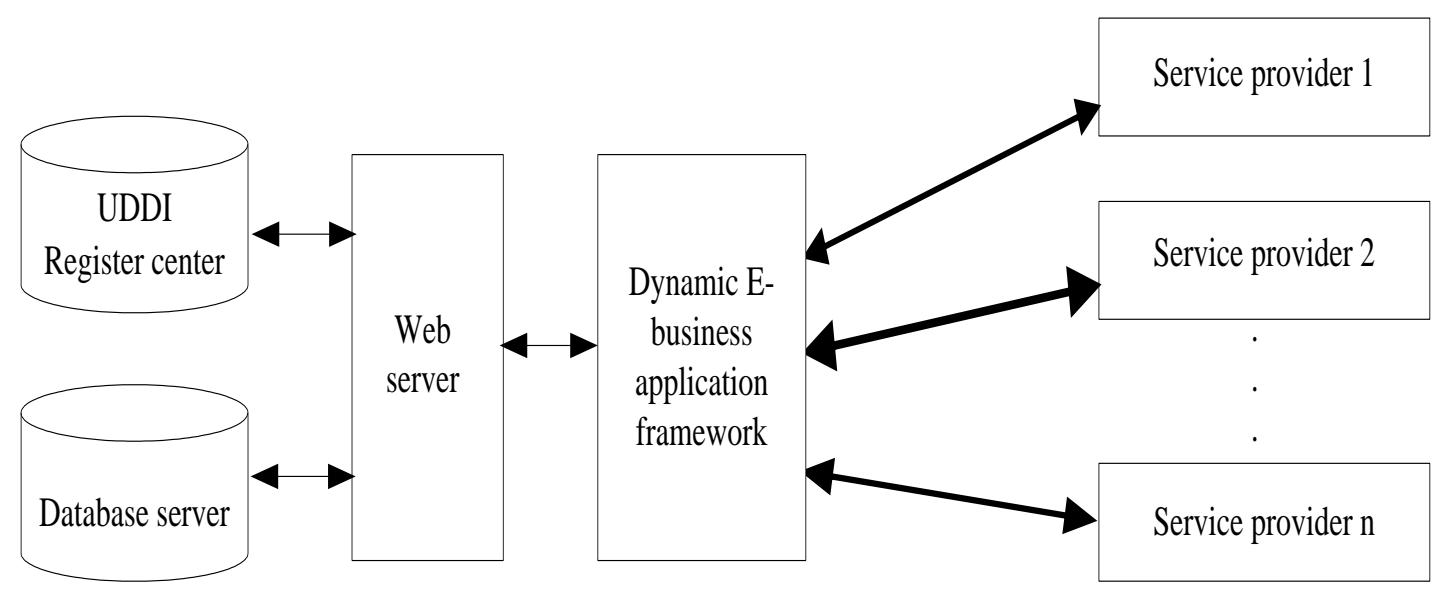

Figure 5. A Novel Dynamic E-Business Application Framework

In proposed dynamic E-business application framework based on Web service-based SOA, each software can be regarded as pellet software component. Web service can receive the request in order to finish a series of specific tasks, then communication standard is used to respond this request for keeping operation. The proposed dynamic Ebusiness application framework based on Web service-based SOA takes on the following characteristics:

\section{(1) The Easy Realization}

The characteristics of Web service determines the easy realization of dynamic Ebusiness application framework based on Web service-based SOA. Many companies (Microsoft, SUN, IBM and so on) provide the massive free tools to fast generate and deploy Web service. And the existing system can easily changed into other system by 
using service. The prototype is realized under Windows.NET framework. This framework is windows component, which is used to construct and run the next generation software application. The framework can support more than 30 kinds of different developing languages, control various insertion operations and conveniently construct, deploy and manage the secure and reliability. The partial related functions are realized by using Form, XML, Web Service, .NET and so on.

\section{(2) The easy integration}

When Web service is published, it will be used to serve according to its introduction by the requested service application. Even if the service is changed, the application framework does not be changed in order to make the integration with other application system.

\section{Conclusion}

The dynamic E-business is the goal of E-business development, and Web service is the kernel and key technology to develop a new dynamic E-business application framework. The dynamic E-business application framework and traditional E-business application framework are expatiated. And SOAP, XML, WSDL and UDDI are the kernel of Web service, which provides open and supporting plug, the independent application of each language and platform for Web service. These technologies promote the dynamic Ebusiness development. And the SOA can meet the requirements of various kinds of Ebusiness application architecture. So a service-oriented dynamic E-business application framework based on Web service-based SOA is proposed. In the proposed dynamic Ebusiness application framework, the SOA is used to realize the standard and loosely coupling application framework. It can ensure a compatible information exchange and integration framework for the business process of the dynamic E-business and provide more complex interaction and collaboration between trading partners. The application framework takes on the characteristics of crossing platform, flexibility and easy expansion.

\section{References}

[1] S. Park, J.M. Choi, H.Y. Yoo, "Integrated model of service-oriented architecture and web-oriented architecture for financial software", Journal of Information Science and Engineering, vol. 28, no. 5, (2012), pp. 925-939

[2] M.Á. Conde, F. García-Peñalvo, M. Alier,E. Mayol, C. Fernández-Llamas. Implementation and design of a service-based framework to integrate personal and institutional learning environments. Science of Computer Programming, 88:41-53 (2014)

[3] G. Flurry, W. Vicknair, "The IBM application framework for e-business. IBM Systems Journal”, vol. 40, no. 1, (2001), pp. 8-24.

[4] K. Rajiv, P. Frank, E. Tim, V. Tom, S. Marylou, B, Gary, "Managing customer relationships through Ebusiness decision support applications: A case of hospital-physician collaboration", Decision Support Systems, vol. 32, no. 2, (2001), pp. 171-187.

[5] W. Hasselbring. Web data integration for E-business applications. IEEE Multimedia, vol. 9, no. 1, (2002), pp. 16-25.

[6] Z.D. Wu, Y. Liu, F.Y. Ma, "Research on service-based e-business application integration framework", Journal of Shanghai Jiaotong University, vol. 36, no. 9, (2002), pp. 1341-1345

[7] H. Li, J.N. Cao, C.L. Daniel, S. Mirosław, "A framework for developing a unified B2B e-trading construction marketplace", Automation in Construction, vol. 12, no. 2, (2003), pp. 201-211

[8] H.M. Al-HelaliAdnan, S.S. Zhang, "Design of the XML security system for electronic commerce application. High Technology Letters", vol. 9, no. 2, (2003), pp. 81-86.

[9] B. Benatallah, F. Casati, F. Toumani, "Web service conversation modeling: A cornerstone for Ebusiness automation", IEEE Internet Computing, vol. 8, no. 1, (2004), pp. 46-54.

[10] Y.H. Cho, J.K. Kim, "Application of Web usage mining and product taxonomy to collaborative recommendations in E-business", Expert Systems with Applications, vol. 26, no. 2, pp. 233-246

[11] H.A. Schmid, G. Rossi, "Modeling and designing processes in E-business applications", IEEE Internet Computing, vol. 8, no. 1, (2004), pp. 19-27. 
[12] D.S. Zhang, M. Chen, L.N. Zhou, "Dynamic and personalized web services composition in E-business", Information Systems Management, vol. 22, no. 3, (2005), pp. 50-65.

[13] G.S. Francisco, V.G. Rafael, M.B. Rodrigo, "An integrated approach for developing E-business applications", Expert Systems with Applications, vol. 28, no. 2, (2005), pp. 223-235.

[14] L.T. March, E.W.T. Ngai. The role of managerial attitudes in the adoption of technological innovations: An application to B2C E-business. International Journal of Enterprise Information Systems, vol. 3, no. 2, (2007), pp. 23-33.

[15] T.H. Chou, Y.M. Lee, "Integrating E-services with a telecommunication E-business using serviceoriented architecture", Journal of Software, vol. 3, no. 9, (2008), pp. 60-67.

[16] Y.S. Li, J.P. Shen, J.S. Shi, W.N. Shen, Y. Huang, Y.X. Xu, "Multi-model driven collaborative development platform for service-oriented e-Business systems", Advanced Engineering Informatics, vol. 22, no. 3, (2008), pp. 328-339.

[17] T.L. Hsia, J.H. Wu, E.Y. Li, "The E-business value matrix and use case model: A goal-driven methodology for eliciting B2C application requirements", Information and Management, vol. 45, no. 5, (2008), pp. 321-330.

[18] J.H. Huang, X.M. Jiang, Q. Tang, “An E-business performance assessment model: Its development and an initial test on E-business applications in the retail sector of China", Information and Management, vol. 46, no. 2, (2009), pp. 100-108.

[19] C.Y. Wei, C. Lv, M. Zhang. Design and implementation of a new integrated E-business system based on AJAX. Journal of Computational Information Systems, vol. 5, no. 2, (2009), pp. 755-762.

[20] C.H. Piao, X.F. Han, H. Wu, "Research on E-business transaction networks using multi-agent modelling and open application programming interface", Enterprise Information Systems, vol. 4, no. 3, (2010), pp. 329-353.

[21] D.V. Meer, K. Dutta, A. Datta, "A cost-based database request distribution technique for online Ebusiness applications”, MIS Quarterly: Management Information Systems, vol. 36, no. 2, (2012), pp. 479-507.

[22] Q.Y. Chen, N. Zhang, "IT-supported business performance and E-business application in SMEs. Journal of Electronic Commerce in Organizations", vol. 11, no. 2, (2013), pp. 41-52

[23] Y.Z. Zhang, R. Mukherjee, B. Soetarman, "Concept extraction and E-business applications", Electronic Commerce Research and Applications, vol. 12, no. 4, (2013), pp. 289-296.

[24] Y.W. Wang, H. Lv, "Study on the influence factors of E-business application on the business model of Chinese real estate enterprises", International Journal of u- and e- Service, Science and Technology, vol. 6, no. 4, (2013), pp. 181-198.

[25] H.H. Gao, H.K. Miao. Research on the dynamic reconfiguration of Web application using two-phase compatibility verification. International Journal of Computer Mathematics, vol. 90, no. 11, (2013), pp. 2265-2278.

[26] Z. Eugenio, O. Gianfranco, B. Fabio, C. Alfonso. Designing a scalable social E-business application. Scalable Computing, vol. 14, no. (2), (2013), pp. 131-141.

[27] J. Liu. Research on the application of E-business in the economic management. Applied Mechanics and Materials, (2014), 687-691:4520-4523.

[28] G.C. Lamprinakos,S. Asanin, T. Broden,A. Prestileo,J. Fursse, K.A. Papadopoulos,D.I. Kaklamani,I.S. Venieris. An integrated remote monitoring platform towards Telehealth and Telecare services interoperability. Information Sciences, 308:23-37(2015)

[29] M.A. Martínez-Carreras, F.J. García Jimenez, A.F. Gómez Skarmeta, "Building integrated business environments: analysing open-source ESB. Enterprise Information Systems”, vol. 9, no. 4, (2015), pp. 401-435.

\section{Author}

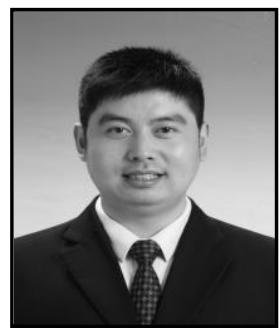

Xueqiang Yin. Senior Engineer, received the Master degree in software engineering, Beijing Jiaotong University in 2015, Beijing, China. The main research directions: Computer Application, Network Communication, Image Algorithm. 
International Journal of Security and Its Applications

Vol.10, No.1 (2016) 